\title{
Simultaneous Chiral Symmetry Restoration and Deconfinement Consequences for the QCD Phase Diagram
}

\author{
Thomas Klähn ${ }^{1}$, Tobias Fischer ${ }^{1}$, and Matthias Hempel ${ }^{2}$ \\ ${ }^{1}$ Institute of Theoretical Physics, University of Wroclaw, Pl. M. Borna 9, 50-204 Wroclaw, Poland; thomas.klaehn@ift.uni.wroc.pl \\ ${ }^{2}$ Department of Physics, University of Basel, Klingelbergstrasse 82, 4056 Basel, Switzerland \\ Received 2016 March 11; revised 2017 January 12; accepted 2017 January 13; published 2017 February 10
}

\begin{abstract}
For studies of quark matter in astrophysical scenarios, the thermodynamic bag model is commonly employed. Although successful, it does not account for dynamical chiral symmetry breaking and repulsions due to the vector interaction which is crucial to explain recent observations of massive, two solar mass neutron stars. In Klähn \& Fischer we developed the novel vBag quark matter model which takes these effects into account. This article extends vBag to finite temperatures and isospin asymmetry. Another particular feature of vBag is the determination of the deconfinement bag constant $B_{\mathrm{dc}}$ from a given hadronic equation of state in order to ensure that chiral and deconfinement transitions coincide. We discuss consequences of this novel approach for the phase transition construction, the phase diagram, and implications for protoneutron stars.
\end{abstract}

Key words: dense matter - elementary particles - equation of state

\section{Introduction}

The theory of strong interactions, i.e., Quantum Chromodynamics (QCD), considers hadrons and mesons as color-neutral compound objects of quarks and gluons. Besides confinement, a second key feature of QCD is dynamical chiral symmetry breaking $(\mathrm{D} \chi \mathrm{SB})$ and its restoration at large densities and high temperatures. Currently, lattice-QCD is the only ab initio approach to solve QCD numerically (cf. Fodor \& Katz 2004; Aoki et al. 2006, and references therein). In the vicinity of vanishing chemical potentials, lattice-QCD predicts a crossover phase transition for both deconfinement and chiral symmetry breaking in the temperature range $150<T<170 \mathrm{MeV}$ (the currently considered value is $154 \pm 9 \mathrm{MeV}$; cf. Bazavov et al. 2012a, 2014, 2012b; Borsányi et al. 2012, 2014). This predicted temperature is in agreement with hadron freeze-out data from heavy-ion collision experiments (cf. Braun-Munzinger et al. 2015 and references therein). However, lattice-QCD calculations are restricted to low chemical potentials. On the other hand, in astrophysical systems, e.g., the interior of neutron stars and core-collapse supernovae, we usually encounter large chemical potentials (or equivalently high densities, even in excess of normal nuclear density), high isospin asymmetries, and finite temperatures. Such conditions are inaccessible by lattice-QCD and the current generation of heavy-ion collision experiments.

The two most commonly used effective quark matter models in astrophysics are the thermodynamic bag model (tdBag) of Farhi \& Jaffe (1984) and models of the Nambu-Jona-Lasinio (NJL) type (cf. Nambu \& Jona-Lasinio 1961; Klevansky 1992; Buballa 2005). Recently, we illustrated in Klähn \& Fischer (2015) that both approaches can be understood as limiting solutions of QCD's in-medium Dyson-Schwinger gap equations within a particular set of approximations. The advantage of the Dyson-Schwinger formalism is a closer connection to QCD in comparison to the aforementioned phenomenological approaches. First explorations of the equation of state $(\mathrm{EoS})$ of deconfined quark matter within this approach have been performed (cf. Chen et al. 2008, 2011, 2015, 2016; Klähn et al. 2010). Perturbative QCD is valid in the limit of asymptotic freedom where quarks are no longer strongly coupled (cf. Kurkela et al. 2014 and references therein). Therefore, it provides a valuable benchmark for the asymptotic behavior of realistic quark matter model EoS.

Currently, no consistent approach exists to describe medium properties of hadrons and mesons at the level of quarks and gluons, in particular at high density. Hence, the deconfinement phase transition is usually constructed from a given hadronic EoS with hadrons and mesons as the fundamental degrees of freedom and an independently computed quark matter EoS. Only few attempts exist to improve this situation; see, e.g., Dexheimer \& Schramm (2010), Steinheimer et al. (2011). Constructions based on Maxwell's conditions by definition result in a first-order phase transition (cf. Rischke et al. 1988; Barz et al. 1989). In Fischer et al. (2011), a Gibbs construction based on tdBag as the quark matter EoS at finite temperatures has been employed for applications in astrophysics. Yasutake et al. (2014) considered pasta structures at the quark-hadron interface and compared the results with standard Maxwell and Gibbs constructions. Further alternative approaches for the construction of possible quark-hadron phase transitions were discussed in Masuda et al. (2013) and Kojo et al. (2015).

In Klähn \& Fischer (2015), we introduced the novel quark matter EoS vBag, in particular for applications in astrophysics. Although vBag is initially motivated as a simplification of an NJL model with scalar and iso-vector condensates, it is essentially an extended bag model that explicitly accounts for the repulsive vector interaction. This property is essential for a stiffening of the EoS toward high densities and results in maximum neutron star masses in agreement with the current constraint of about $2 M_{\odot}$ from the observations of Antoniadis et al. (2013) and Demorest et al. (2010), the latter recently revised to $1.928 \pm 0.017 M_{\odot}$ by Fonseca et al. (2016). Moreover, vBag mimics (de)confinement via a phenomenological pressure correction to the EoS in the form of a deconfinement bag constant $B_{\mathrm{dc}}$. The simultaneous occurrence of chiral symmetry restoration and (de)confinement is generally not provided for a "standard" Maxwell or Gibbs construction. This represents an essential assumption for this study. The consequences will be discussed in detail. The idea to enforce 
deconfinement at the chiral transition by manually equating the pressure of hadronic and quark matter EoS at zero temperature has been discussed previously by Pagliara \& SchaffnerBielich (2008).

In this article we extend vBag to finite temperatures and isospin asymmetry. As a consequence of our model prescription, the deconfinement bag constant $B_{\mathrm{dc}}$ becomes medium dependent and depends directly on the hadronic EoS. The medium dependence induces correction terms for the EOS of the quark phase, which consequently depends on the hadronic EOS as well. For illustration, we select a nuclear EoS from the catalog of Hempel \& Schaffner-Bielich (2010). It treats the nuclear medium within a quasi-particle picture of the relativistic mean-field (RMF) framework for conditions in excess of normal nuclear matter density and high temperatures. Specifically, we choose the parametrization DD2 from Typel et al. (2010). We aim to make vBag accessible to a broader spectrum of astrophysics applications where the appearance of quark matter can be studied, e.g., simulations of neutron star mergers, core-collapse supernovae, and protoneutron star cooling (cf., Pons et al. 2001; Nakazato et al. 2008; Sagert et al. 2009; Fischer et al. 2011).

In Section 2 we review the basic equations of vBag, including novel aspects due to the extension to finite temperature and isospin asymmetry. In Section 3 we construct the hybrid EoS HS(DD2)-vBag based on a quark matter EoS parametrization that was already used in Klähn \& Fischer (2015). We describe the resulting phase diagram and differences from the standard approach for the construction of the phase transition. In Section 4 we discuss possible astrophysical applications using the example of protoneutron stars. Section 5 is devoted to limitations of this study with a detailed comparison of vBag and NJL. The manuscript closes with a summary in Section 6.

\section{2. vBag as a Hybrid Approach}

\subsection{Chiral Symmetry and Vector Interaction}

In Klähn \& Fischer (2015), we introduced the vBag model as an extension of tdBag, which in turn was introduced in Farhi \& Jaffe (1984). As is typical for quasi-particle approaches, the thermodynamical behavior of vBag is fully expressed in terms of non-interacting Fermi gas integrals. The model considers only the chirally restored phase where the quark mass is well approximated by the bare quark mass. Despite this similarity to the standard bag model, vBag accounts explicitly for the repulsive vector interaction and the dynamical breaking of chiral symmetry at a critical chemical potential $\mu_{\chi, f}$, where $f$ denotes the quark flavor.

For $\mu_{f}<\mu_{\chi, f}$, chiral symmetry is broken and we assume that quarks are confined in hadron and meson states that are not accessible to vBag. The chiral phase transition and the corresponding critical chemical potential $\mu_{\chi, f}$ is defined by the value of the chiral bag constant $B_{\chi}$, demanding that at $\mu_{f}=\mu_{\chi, f}$, the pressure of a single quark flavor, which is given as

$$
P_{f}\left(\mu_{f}\right)=P_{\mathrm{FG}, f}^{\mathrm{kin}}\left(\mu_{f}^{*}\right)+\frac{K_{v}}{2} n_{\mathrm{FG}, f}^{2}\left(\mu_{f}^{*}\right)-B_{\chi, f},
$$

turns positive (for illustrations cf. Figure 1 of Klähn \& Fischer 2015). $B_{\chi, f}$ is a flavor-dependent parameter that can be computed from a microscopic approach as the difference between the vacuum pressure of the chirally broken and the chirally restored phase.

As this prescription is applied to each flavor independently, it would result in sequential chiral transitions of light and heavy quarks (more details are given in Klähn \& Fischer 2015). We return to this point during the discussion of the phase transition in Section 2.2.

The constant $K_{v}$ in the second term in Equation (1) relates directly to the coupling strength $G_{v}$ of the vector currentcurrent interaction as one would define it in NJL type models. As explained in more detail in Klähn \& Fischer (2015), $B_{\chi, f}$ (besides the regularization scheme and involved parameters) depends on a corresponding scalar coupling constant $G_{s}$, which itself is related to $G_{v}$ by $G_{v}=G_{s} / 2$. Hence, $K_{v}$ and $B_{\chi, f}$ are not strictly independent quantities. However, as the latter relation is not expected to hold strictly in a realistic model, we consider these two as rather independent model parameters of vBag. A consequence of accounting for the vector interaction that leads beyond the tdBag model is the appearance of the effective chemical potential $\mu_{f}^{*}$ which enters all Fermi gas expressions. The actual chemical potential $\mu_{f}$ in the sense of a thermodynamic variable is determined post priori as

$$
\mu_{f}=\mu_{f}^{*}+K_{v} n_{\mathrm{FG}, f}\left(\mu_{f}^{*}\right) .
$$

This again is common for quasi-particle models where interactions dynamically alter particle in-medium properties, e.g., mass and chemical potential but not the structure of the Fermi integrals.

With Equations (1) and (2), the EoS at zero temperature is given and the two relations can be used to determine the energy density and quark number density of a given flavor:

$$
\begin{gathered}
\varepsilon_{f}\left(\mu_{f}\right)=\varepsilon_{\mathrm{FG}, f}^{\mathrm{kin}}\left(\mu_{f}^{*}\right)+\frac{K_{v}}{2} n_{\mathrm{FG}, f}^{2}\left(\mu_{f}^{*}\right)+B_{\chi, f}, \\
n_{f}\left(\mu_{f}\right)=n_{\mathrm{FG}, f}\left(\mu_{f}^{*}\right) .
\end{gathered}
$$

To extend the model to finite temperatures, we replace the zero temperature Fermi gas integrals by the equivalent finite temperature expressions. The entropy density of the system is expressed in the quasi-particle picture again:

$$
s_{f}\left(T, \mu_{f}\right)=\left.\frac{\partial P_{f}\left(T, \mu_{f}\right)}{\partial T}\right|_{\mu_{f}}=s_{\mathrm{FG}, f}\left(T, \mu_{f}^{*}\right) .
$$

One easily verifies the Euler equation $\varepsilon+P=\mu n+T s$ to be fulfilled. Notice that in this study we assume a constant, medium-independent $B_{\chi, f}$. This is not a perfect or in general correct choice - the scalar condensate is known to melt with increasing temperature-and we assume for the moment not to reach high enough temperatures to induce significant changes of the pressure due to this melting. We briefly discuss the limitations of this assumption in Section 5.

\subsection{Deconfinement}

The previous section described how vBag accounts for the repulsive vector interaction and the chiral phase transition of a single quark flavor. These results can be perfectly understood in terms of a simple NJL model or more generally in the framework of Dyson-Schwinger model studies assuming an underlying gluon contact interaction as illustrated in Klähn \& Fischer (2015). vBag is a limit of these models in the chirally 
restored phase, assuming that in this domain the bare quark mass is a sufficient approximation of the real mass gap solutions to describe the thermodynamical behavior of chirally restored quark matter. The condition $P_{f}\left(\mu_{\chi, f}\right)=0$, which determines $\mu_{\chi, f}$, does not imply that the chiral bag constant describes the pressure under a confining force but simply accounts for the fact that chiral symmetry in confined matter is broken, and subsequently, the restoration of chiral symmetry induces pressure in the system.

If one aims to account for both chiral symmetry restoration and deconfinement, it is worth considering the following two aspects. First, a description of hadronic matter that does not take chiral symmetry breaking on the quark level into account is likely inaccurate. It is flawed already on the level of the "fundamental" bare hadron masses independently from the specific modeling of nucleon-nucleon interactions. Second, hadronic matter will deconfine after the system reaches a sufficient critical chemical potential $\mu_{B, \mathrm{dc}}$ and related pressure. It is a common approach to describe hybrid nuclear-quark EoS including the chiral/deconfinement transition without giving any of these two statements too much thought by performing a Maxwell or Gibbs construction to connect nuclear and quark EoS; both EoS can be-and in astrophysical applications usually are-obtained from different, independent models.

With these considerations in mind we model a hybrid, nuclear-light-quark EoS for applications at finite temperature and arbitrary isospin asymmetry. While an extension to finite temperatures is easily achieved, isospin asymmetry, i.e., $\mu_{u} \neq \mu_{d}$, raises the problem that different light quark chemical potentials imply situations where, e.g., $\mu_{d}<\mu_{\chi,(u, d)}<\mu_{u}$, and consequently $u$-quarks are chirally restored while $d$-quarks are not. This can lead to interesting scenarios (cf. Blaschke et al. 2009). However, in this work we model an EoS where light flavors appear at the same critical baryon chemical potential analogous to the results obtained with the "standard" approach for first-order phase transitions. To achieve this, we redefine the chiral threshold condition to hold for the total light quark pressure instead of independently for each single flavor:

$$
\sum_{f=u, d} P_{f}\left(T, \mu_{\chi, f}\right)=0 .
$$

Here, $P_{f}\left(T, \mu_{f}\right)$ is defined as the total quark pressure of a single quark flavor according to Equation (1). Based on the NJL model parameterization (Grigorian 2007) we used in Klähn \& Fischer (2015), we assume that the threshold density for the appearance of the heavy strange quark at zero temperature is larger than the light quark threshold. At least in this domain it will not affect the deconfinement bag constant. We omit possible contributions at finite temperature. To be consistent, we neglect hyperon contributions to the hadronic EoS. We point out that technically, our prescription remains the same if one would consider strangeness. This additional degree of freedom is likely to affect the quantitative results of this study but not the prescription we suggest in this work to obtain a medium-dependent deconfinement bag constant and the related correction terms to the EoS.

It is useful to introduce the baryon and charge chemical potentials for light quark matter, which follow in general from $\mu_{f}=\mathcal{B}_{f} \mu_{B}+\mathcal{Q}_{f} \mu_{C}$, with $\mathcal{B}_{f}$ and $\mathcal{Q}_{f}$ being the baryon and electric charge number of the corresponding quark flavor $f$.
This allows us to rewrite

$$
\begin{gathered}
\mu_{B}=\mu_{u}+2 \mu_{d}, \\
\mu_{C}=\mu_{u}-\mu_{d} .
\end{gathered}
$$

At a given $T$ and $\mu_{C}$, we denote our critical chemical potentials for $\mathrm{D} \chi \mathrm{SB}$ and confinement as $\mu_{B, \chi}$ and $\mu_{B, \mathrm{dc}}$, respectively. The general thermodynamic identities,

$$
\frac{\partial P}{\partial \mu_{B}}=n_{B}, \frac{\partial P}{\partial \mu_{C}}=n_{C}, \frac{\partial P}{\partial T}=s,
$$

relate the pressure derivatives with respect to the baryonic and charge chemical potentials to the baryon and charge number densities as well as the temperature derivative to the entropy.

In this work, we assume that at sufficiently low temperatures, the assumption of a first-order phase transition is correct. The condition for the transition from hadronic $(\mathrm{H})$ to quark matter (Q) for the total pressure of each phase reads $P^{\mathrm{H}}\left(\mu_{B, \mathrm{dc}}\right)=P^{\mathrm{Q}}\left(\mu_{B, \mathrm{dc}}\right)$ at a given $T$ and $\mu_{C}$. It defines the deconfinement baryonic chemical potential $\mu_{B, \mathrm{dc}}$. We observe that (i) evidently, deconfinement occurs at finite pressure and (ii) without further modifications, Equation (6) enforces zero pressure at the chiral transition. Hence, chiral and deconfinement transitions are necessarily located at different chemical potentials, $\mu_{B, \chi}<\mu_{B, \mathrm{dc}}$. In fact, the gap between these critical chemical potentials easily spans several $100 \mathrm{MeV}$. The gap region in this two-phase approach is obtained by comparing a nuclear matter EoS that does not know about chiral symmetry breaking on the quark level with a quark matter EoS that does not know about confinement. The thermodynamically favored solution in this domain is the hadron EoS due to its higher pressure, and hence the chiral restoration of quark matter is effectively postponed ad hoc until $\mu_{B, \mathrm{dc}}$ is reached. ${ }^{3}$ With vBag we could model the same late onset of deconfinement. However, to maintain our assumption of simultaneous chiral symmetry restoration and deconfinement, this would require shifting $\mu_{B, \chi}$ and hence increasing the chiral bag constant to higher values, which are not in agreement with, e.g., the NJL model.

We go the opposite way and want our model to account for simultaneous chiral symmetry breaking and the confinement transition at $\mu_{B, \chi}$. We emphasize that this is an assumption we put into this model and not a general property of vBag. According to this idea, $\mu_{B, \mathrm{dc}}$ has to decrease in order to match $\mu_{B, \chi}$, in agreement with Equation (6). This is achieved by exploiting the fact that the total pressure is fixed by the previous equations only up to a constant $B_{\mathrm{dc}}$,

$$
P^{\mathrm{Q}}=\sum_{f} P_{f}\left(T, \mu_{f}\right)+B_{\mathrm{dc}} .
$$

Introducing $B_{\mathrm{dc}}$ thus enables us to ensure that the pressure of hadronic and quark matter is equal at the critical chemical potential $\mu_{B, \chi}$ for the chiral transition. In order to match both pressures, one easily finds the value of $B_{\mathrm{dc}}=P^{\mathrm{H}}\left(\mu_{B, \chi}\right)$ at a given $T$ and $\mu_{C}$. In general, we expect $B_{\mathrm{dc}}$ to depend on all thermodynamic variables, $T, \mu_{C}$, and $\mu_{B}$. However, our

\footnotetext{
3 Note how this picture differs from that of the quarkyonic phase (cf., McLerran \& Pisarski 2007; McLerran et al. 2009; Andronic et al. 2010), where chiral symmetry is restored in the confined domain, $\mu_{B, \chi}<\mu_{B}<\mu_{B, \mathrm{dc}}$ Strictly, this would require reformulating the nuclear EoS to accoun dynamically for nucleon masses, which depend on medium-dependent quarkconstituent masses.
} 
prescription fixes $B_{\mathrm{dc}}$ only on a hypersurface within this threedimensional parameter space, which leaves one free variable and the corresponding dependence of $B_{\mathrm{dc}}$ remains undetermined. We eliminate this degree of freedom by assuming $B_{\mathrm{dc}}\left(T, \mu_{C}, \mu_{B}\right)=B_{\mathrm{dc}}\left(T, \mu_{C}, \mu_{B, \chi}\right)$, where $\mu_{B, \chi}$ itself is a function of $T$ and $\mu_{C}$.

\subsection{Medium Corrections}

We emphasize that we assume that chiral symmetry breaking and deconfinement are tied to values of the corresponding critical chemical potentials that are the same or similar. Whether this will be supported by future research or not, the previous paragraph outlined that both approaches we described imply $\mu_{B, \chi}=\mu_{B, \mathrm{dc}}$ : the traditional phase transition construction of two independent phases by shifting $\mu_{B, \chi} \rightarrow \mu_{B, \mathrm{dc}}$ and the $B_{\mathrm{dc}}$ approach by shifting $\mu_{B, \mathrm{dc}} \rightarrow \mu_{B, \chi}$. We prefer the latter as it keeps the insights from a chiral description on the quark level, and as we show in the following, results in an inherent connection of nuclear and quark EoS that goes beyond the "standard" two-phase approach. Note that vBag does not require this procedure, and in principle we could obtain any value for $\mu_{B, \mathrm{dc}}$ with a corresponding choice of $B_{\mathrm{dc}}$. In fact, the prescribed procedure can be applied to any quark model that accounts for $\mathrm{D} \chi \mathrm{SB}$ and is not restricted to vBag. However, the problem of an existing domain where the favored nuclear EoS is blind to the restoration of chiral symmetry on the quark level would still stand.

Ignoring the vector interaction-induced terms for the moment, vBag behaves very similarly to the tdBag model as it results in an effective bag constant

$$
B_{\text {eff }}=\sum_{f=u, d} B_{\chi \cdot f}-B_{\mathrm{dc}},
$$

which takes similar values to familiar one from the bag model. We emphasize that unlike the standard bag model, in vBag the positive value of $B_{\text {eff }}$ originates from $\mathrm{D} \chi \mathrm{SB}$ only while confinement reduces it. In this sense, $B_{\mathrm{dc}}$ is understood as a confining binding energy. Moreover, $B_{\text {eff }}$ can take values smaller than those in the tdBag model without predicting deconfined (light)-quark matter to be energetically favored over nuclear matter. This is explained in more detail in Klähn \& Fischer (2015).

As outlined earlier, the deconfinement bag constant $B_{\mathrm{dc}}$ depends on the nuclear EoS. According to our prescription for the phase transition, it will vary with temperature and asymmetry, $B_{\mathrm{dc}} \rightarrow B_{\mathrm{dc}}\left(T, \mu_{C}\right)$, while we keep it constant with respect to $\mu_{B}$. This medium dependence of $B_{\mathrm{dc}}$ results in additional contributions to the charge number and entropy density,

$$
\begin{gathered}
n_{C}^{\mathrm{Q}}\left(T, \mu_{C}, \mu_{B}\right)=\tilde{n}_{C}^{\mathrm{Q}}\left(T, \mu_{C}, \mu_{B}\right)+\frac{\partial B_{\mathrm{dc}}}{\partial \mu_{C}}, \\
s^{\mathrm{Q}}\left(T, \mu_{C}, \mu_{B}\right)=\tilde{s}^{\mathrm{Q}}\left(T, \mu_{C}, \mu_{B}\right)+\frac{\partial B_{\mathrm{dc}}}{\partial T},
\end{gathered}
$$

with $\tilde{n}_{C}^{\mathrm{Q}}=\sum_{f=u, d} \mathcal{Q}_{f} n_{f}=\frac{1}{3}\left(2 n_{u}-n_{d}\right)$ and $\tilde{s}^{\mathrm{Q}}=\sum_{f=u, d} s_{f}$. The derivative terms in Equations (12) and (13) distinguish our model from tdBag and other models with the "standard" phase transition construction of two independent phases. One obtains

$$
\begin{aligned}
\frac{\partial B_{\mathrm{dc}}}{\partial \mu_{C}}= & n_{C, \mathrm{dc}}\left(T, \mu_{C}\right) \\
= & \frac{\partial}{\partial \mu_{C}}\left\{P^{\mathrm{H}}\left(T, \mu_{C}, \mu_{B}=\mu_{B, \chi}\left(T, \mu_{C}\right)\right)\right\} \\
= & \frac{\partial P^{\mathrm{H}}}{\partial \mu_{C}}\left(T, \mu_{C}, \mu_{B, \chi}\right)+\frac{\partial \mu_{B, \chi}}{\partial \mu_{C}} \frac{\partial P^{\mathrm{H}}}{\partial \mu_{B}}\left(T, \mu_{C}, \mu_{B, \chi}\right) \\
= & n_{C}^{\mathrm{H}}\left(T, \mu_{C}, \mu_{B, \chi}\right)-\tilde{n}_{C}^{\mathrm{Q}}\left(T, \mu_{C}, \mu_{B, \chi}\right) \\
& \times \frac{n_{B}^{\mathrm{H}}\left(T, \mu_{C}, \mu_{B, \chi}\right)}{n_{B}^{\mathrm{Q}}\left(T, \mu_{C}, \mu_{B, \chi}\right)}, \\
\frac{\partial B_{\mathrm{dc}}}{\partial T}= & : s_{\mathrm{dc}}\left(T, \mu_{C}\right) \\
= & \frac{\partial}{\partial T}\left\{P^{\mathrm{H}}\left(T, \mu_{C}, \mu_{B}=\mu_{B, \chi}\left(T, \mu_{C}\right)\right)\right\} \\
= & \frac{\partial P^{\mathrm{H}}}{\partial T}\left(T, \mu_{C}, \mu_{B, \chi}\right)+\frac{\partial \mu_{B, \chi}}{\partial T} \frac{\partial P^{\mathrm{H}}}{\partial \mu_{B}}\left(T, \mu_{C}, \mu_{B, \chi}\right) \\
= & s^{\mathrm{H}}\left(T, \mu_{C}, \mu_{B, \chi}\right)-\tilde{s}^{\mathrm{Q}}\left(T, \mu_{C}, \mu_{B, \chi}\right) \\
& \times \frac{n_{B}^{\mathrm{H}}\left(T, \mu_{C}, \mu_{B, \chi}\right)}{n_{B}^{\mathrm{Q}}\left(T, \mu_{C}, \mu_{B, \chi}\right)} .
\end{aligned}
$$

At $\mu_{C}=0$ one has isospin symmetric matter and hence $Y_{C}^{\mathrm{H}}=\tilde{Y}_{C}^{\mathrm{Q}}=0.5$ (with the charge fractions $Y_{C}$ defined as $\left.n_{C} / n_{B}\right)$, which implies $n_{C, \mathrm{dc}}\left(T, \mu_{C}=0\right)=0$. Similarly at $T=0, s^{\mathrm{H}}=\tilde{s}^{\mathrm{Q}}=0$ holds and thus $s_{\mathrm{dc}}\left(T=0, \mu_{C}\right)=0$. It is evident that the definition of $B_{\mathrm{dc}}\left(T, \mu_{C}\right):=P_{\mathrm{H}}\left(T, \mu_{C}, \mu_{B, \chi}\right)$ entangles vBag with the hadron EoS (labeled $\mathrm{H}$ ). In this very aspect the finite temperature and isospin asymmetry extension of $\operatorname{vBag}\left(T, \mu_{C}\right)$ differs from the $T=0 \mathrm{vBag}$ case discussed in Klähn \& Fischer (2015). The procedure does not induce a deconfinement baryon density, i.e., $n_{B}^{\mathrm{Q}}=\tilde{n}_{B}^{\mathrm{Q}}=\sum_{f=u, d} \mathcal{B}_{f} n_{f}=$ $\frac{1}{3}\left(n_{u}+n_{d}\right)$, one has to take corrections to the energy density into account,

$$
\begin{aligned}
\varepsilon^{\mathrm{Q}}\left(T, \mu_{C}, \mu_{B}\right)= & \tilde{\varepsilon}^{\mathrm{Q}}\left(T, \mu_{C}, \mu_{B}\right)-B_{\mathrm{dc}}\left(T, \mu_{C}\right) \\
& +T s_{\mathrm{dc}}\left(T, \mu_{C}\right)+\mu_{C} n_{C, \mathrm{dc}}\left(T, \mu_{C}\right),
\end{aligned}
$$

with $\tilde{\varepsilon}^{\mathrm{Q}}=\sum_{f=u, d} \varepsilon_{f}$. We name the corrections terms as the deconfinement energy density, $\varepsilon_{\mathrm{dc}}\left(T, \mu_{C}\right)=T s_{\mathrm{dc}}\left(T, \mu_{C}\right)+$ $\mu_{C} n_{C, \mathrm{dc}}\left(T, \mu_{C}\right)$.

\section{Phase Diagram}

Figure 1 illustrates the difference of our approach from the "standard" phase transition construction. We choose a point in the phase diagram $\left(\mu_{B}=1125 \mathrm{MeV}, \mu_{C}=-150 \mathrm{MeV}\right.$, $T=30 \mathrm{MeV}$ ) and examine the pressure dependence to changes of one of these variables. In general, the vector coupling is set to zero except for set (a), where we show two cases, $K_{v}=0$ and $K_{v}=2 \times 10^{-6} \mathrm{MeV}^{-2}$. Note that only quarks, neutrons, and protons are considered-leptons, further baryons, and purely thermal excitation states, e.g., photons, pions, and gluons, are not taken into account. For the purpose of 
(a)

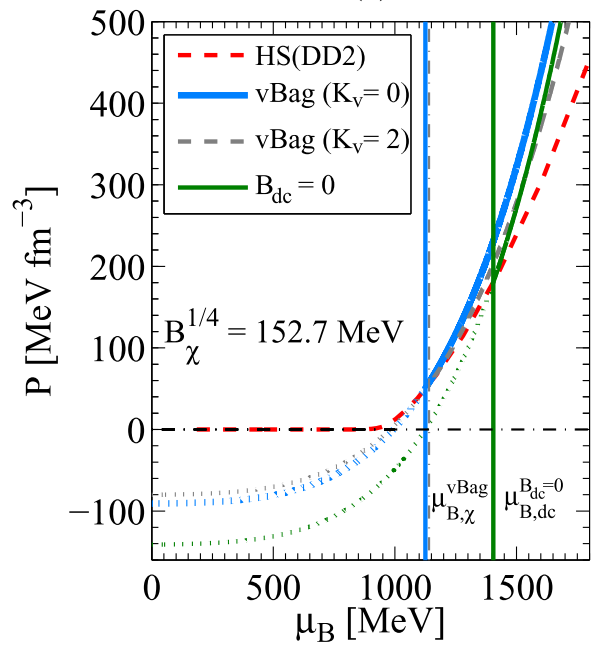

$\left(\mathrm{T}=30 \mathrm{MeV}, \mu_{\mathrm{C}}=-150 \mathrm{MeV}\right)$

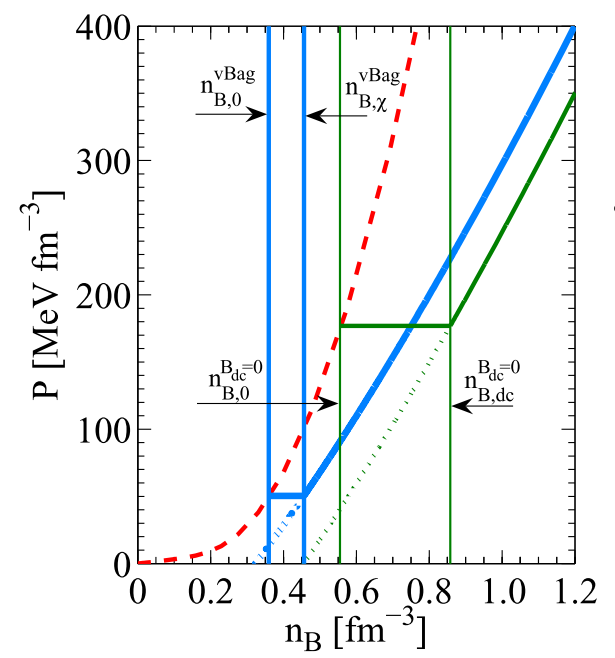

(b)

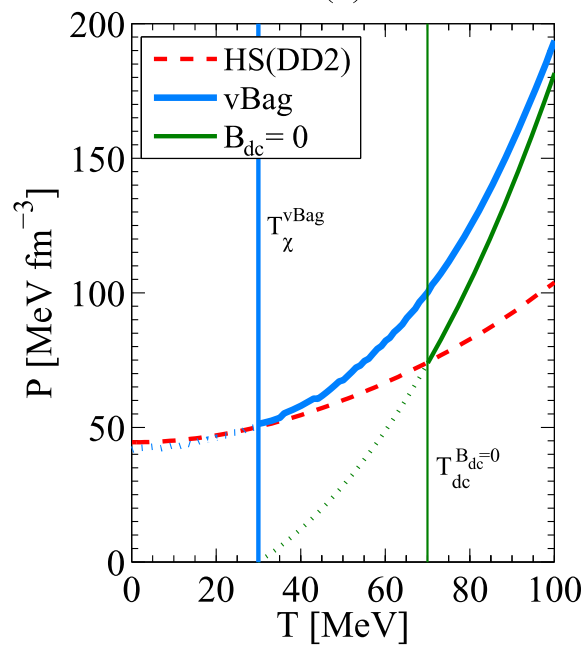

$\left(\mu_{\mathrm{B}}=1125 \mathrm{MeV}, \mu_{\mathrm{C}}=-150 \mathrm{MeV}\right)$

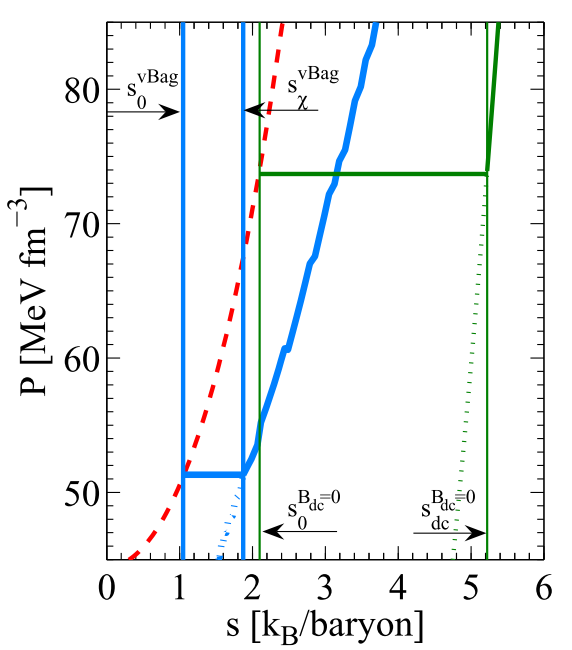

(c)

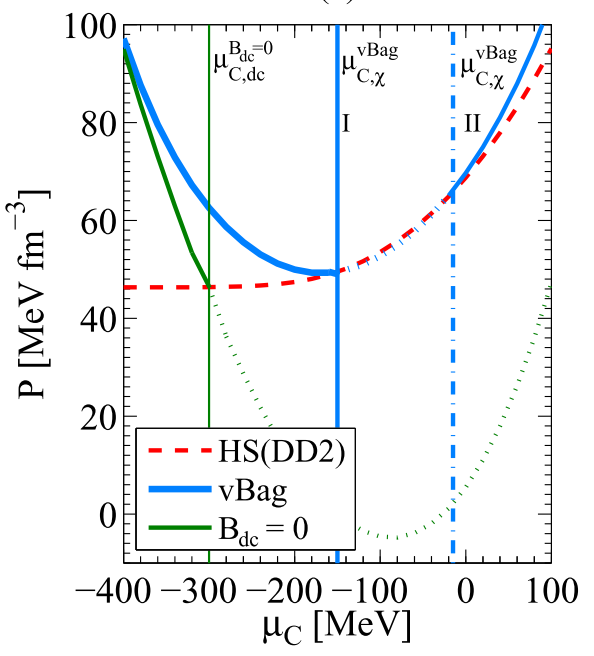

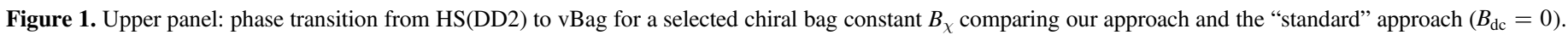

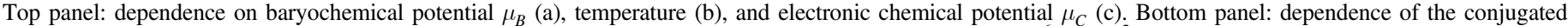
thermodynamical variable (see text for definitions). For (a), zero and finite vector coupling $K_{v}$ (units of $10^{-6} \mathrm{MeV}^{-2}$ ) are considered.

illustration, we chose the nuclear HS(DD2) EoS from Hempel \& Schaffner-Bielich (2010) based on the RMF parametrization DD2 from Typel et al. (2010) as it covers finite baryon number density, temperature, and isospin asymmetry. It is available at the CompOSE database. ${ }^{4}$

We refer to the "standard" phase transition construction as a prescription that takes the EoS of both phases as independent input and favors the phase with higher pressure at given chemical potential. Such a model is represented by the green line in Figure 1, where the light quark single flavor chiral bag constant has a value of $B_{\chi}^{1 / 4}=152.7 \mathrm{MeV}$ (see Table 1 in Klähn \& Fischer 2015) while we set $B_{\mathrm{dc}} \equiv 0$. With this setup we reproduce the underlying NJL model at zero temperature. At small temperatures, the model predicts the restoration of chiral symmetry at $\mu_{B, \chi} \approx 1150 \mathrm{MeV}$ defined by the condition $P^{\mathrm{Q}}\left(\mu_{B, \chi}, \mu_{C}, T\right)=0$. However, deconfinement as the transition from nuclear to quark matter occurs at a higher chemical potential (as $\left.P^{\mathrm{H}}\left(\mu_{B, \mathrm{dc}}\right)=P^{\mathrm{Q}}\left(\mu_{B, \mathrm{dc}}\right)>0\right)$, in this particular

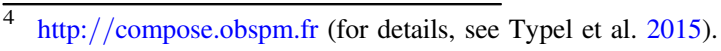

case at $\mu_{B, \mathrm{dc}} \approx 1400 \mathrm{MeV}$. For all $\mu_{B}<\mu_{B, \mathrm{dc}}, \mathrm{HS}(\mathrm{DD} 2)$ is determined as the thermodynamically favored EoS, although the quark matter model already predicts the restoration of chiral symmetry on the quark level at a significantly lower value. The restoration of chiral symmetry is by construction not accounted for by HS(DD2).

In our approach, we mimic confinement by introducing $B_{\mathrm{dc}}\left(T, \mu_{C}\right)=P^{\mathrm{H}}\left(T, \mu_{C}, \mu_{B}=\mu_{B, \chi}\right)$ as a positive shift of the quark pressure as discussed in the previous section, and therefore (i) lower the onset of deconfinement $\mu_{B, \mathrm{dc}} \rightarrow \mu_{B, \chi}$ and (ii) do not favor the nuclear EoS in a region where the dressed nucleon mass due to $\mathrm{D} \chi \mathrm{SB}$ should be lower than HS(DD2) accounts for. The result of our approach is illustrated by the solid blue line in Figure 1. As we wish to study the consequences of our approach for the QCD phase transition in the phase diagram, we ignore the influence of vector coupling-dependent terms in our model. The reason is easily understood from the red dashed line in the same figure. Here we chose a typical value for the vector coupling constant, $K_{v}=2 \times 10^{-6} \mathrm{MeV}^{-2}$. Although the thermodynamic correction terms induced by the vector interaction do visibly play an 
important role at higher densities, they have an almost negligible influence on the position of $\mu_{B, \chi}$ and the thermodynamical quantities at this value.

The lower-left panel of Figure 1 translates the chemical potential from the upper-left panel into the corresponding baryon number densities. Both the onset baryon densities of the phase transition $\left(n_{B, 0}\right)$ and pure quark matter $\left(n_{B, \chi}\right)$ are shifted to smaller densities, and the phase coexistence region $\left[n_{B, 0}, n_{B, \chi}\right]$ shrinks in comparison to the standard approach. Our construction implies that $n_{B, \chi}$ depends only on the chiral quark model $\left(n_{B, \chi}\right.$ is the slope of $P^{\mathrm{Q}}\left(\mu_{B}\right)$ with respect to $\mu_{B}$ at $\mu_{B, \chi} . B_{\mathrm{dc}}$ does not depend on $\mu_{B}$ and consequently does not contribute to $n_{B}$.), while $n_{B, 0}$ is sensitive to both the nuclear and quark matter EoS, as $\mu_{B, \mathrm{dc}}=\mu_{B, \chi}$ and $n_{B, 0}=n_{B}^{\mathrm{H}}\left(\mu_{B, \mathrm{dc}}\right)$. We consider the effect dramatic. Whereas the "standard" approach predicts the onset of the phase transition at about $3.5 n_{S}$, our approach shifts the onset close to $2.5 n_{S}$ (the saturation density for HS(DD2) is $n_{S}=0.155 \mathrm{fm}^{-3}$ ). Further, the density range covered by the phase coexistence region (visible as a plateau in the lower panel of Figure 1) is reduced from $2 n_{S}$ to less than $0.5 n_{s}$. Qualitatively, we find this shift of the phase transition toward smaller values for all thermodynamic degrees of freedom $\left(\mu_{B}, \mu_{C}\right.$, and $\left.T\right)$ together with a narrowing of the transition region in terms of the conjugated quantities $\left(n_{B}^{\mathrm{Q}}, n_{C}^{\mathrm{Q}}\right.$, $\left.s^{\mathrm{Q}}\right)$. This is illustrated in the remaining panels of Figure 1 . Although the phase transition along the temperature axis (b) behaves qualitatively similar to what we observe in the dependence on the baryochemical potential (a) we notice a sequence of two phase transitions (quark to hadron (I) and hadron to quark (II)) along the axis of the electronic chemical potential in column (c).

We further illustrate the differences between our and the "standard" approach by showing the phase boundary at finite temperatures and chemical potentials for two selected values of the charge chemical potential $\left(\mu_{C}=(-200,0) \mathrm{MeV}\right)$ in Figure 2. As is expected, $\mu_{B, \chi}$ decreases with increasing temperature. Moreover, our approach suggests a widening of the phase transition region in terms of density with increasing temperature while the "standard" approach describes a narrowing. This is related to the different temperature behaviors of HS(DD2) and vBag, with a faster softening for the hadronic EoS. In our approach, the upper border of the instability region (solid black and red lines in panel (b)) is exclusively defined by the chiral transition line predicted by vBag. In the standard approach, the restoration of chiral symmetry is explicitly ignored. Unlike vBag, the upper border of the transition region is influenced by the underlying nuclear matter EoS, which together with the quark EoS determines the location of the phase transition. In comparison to the differences due to a different modeling of the phase transition, only small variations in terms of $\mu_{B, \chi}$ and $\rho_{\chi}$ are found for varying $K_{v}$ (see Figure 1) or the isospin asymmetry (Figure 2).

The significant changes of the location and width of the phase transition region are a direct result of the introduction of the medium-dependent deconfinement bag constant $B_{\mathrm{dc}}$. As stated earlier, it maps the nuclear EoS at $\mu_{B, \chi}$ in order to align the chiral and deconfinement phase transition. The critical chemical potential for the chiral transition depends on the particular value of the chiral bag constant $B_{\chi}$, the temperature $T$, and the isospin asymmetry. In Figures 3 and 4 , we illustrate the dependence of $B_{\mathrm{dc}}$ on temperature and charge chemical

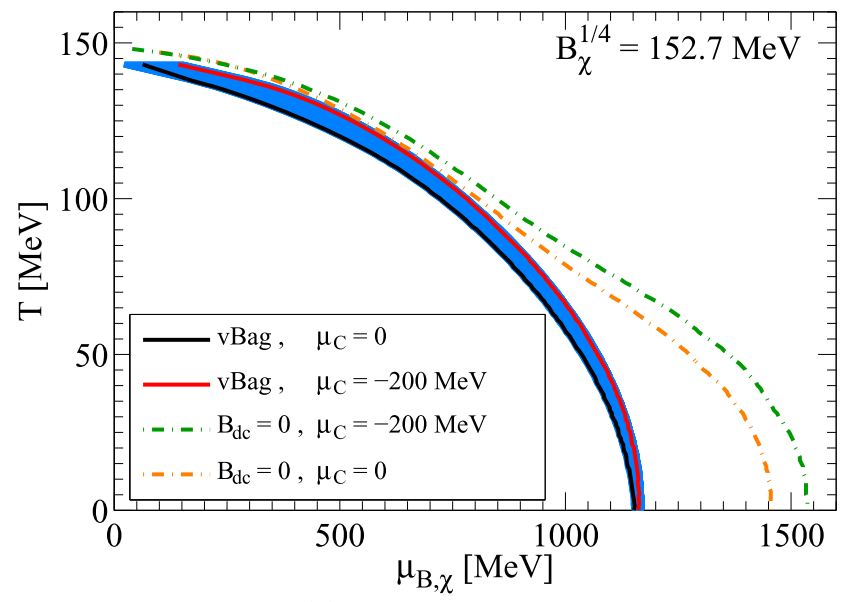

(a) $T-\mu_{B}$ plane

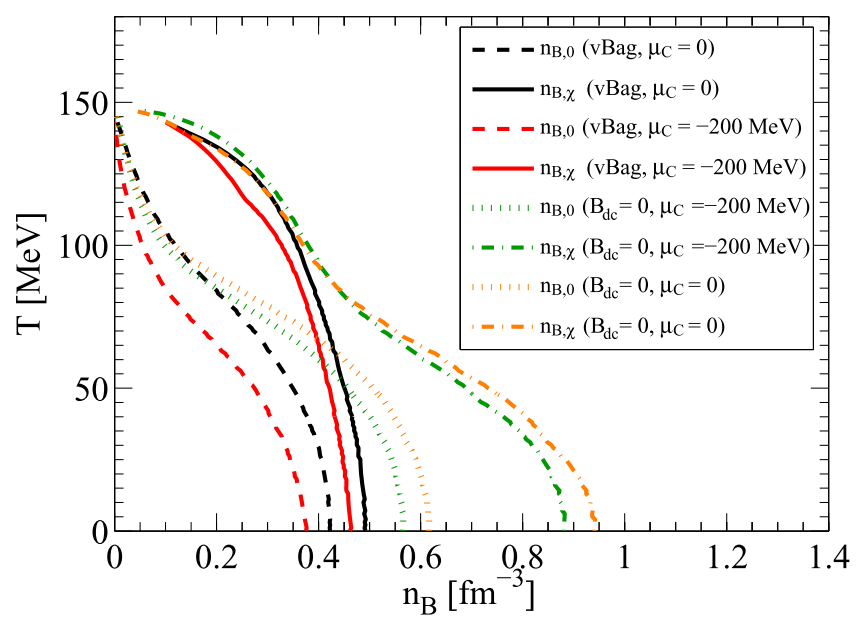

(b) $T-n_{B}$ plane

Figure 2. Upper panel: phase diagram for the transition from HS(DD2) to vBag with a selected single flavor chiral bag constant, $B_{\chi}^{1 / 4}=152.7 \mathrm{MeV}$ for $\mu_{C}=0$ (symmetric matter) and $\mu_{C}=-200 \mathrm{MeV}$ (strongly asymmetric matter) comparing our and the "standard" approach $\left(B^{\mathrm{dc}}=0\right)$. Bottom panel: corresponding density dependence of the phase diagram (see the text for definitions).

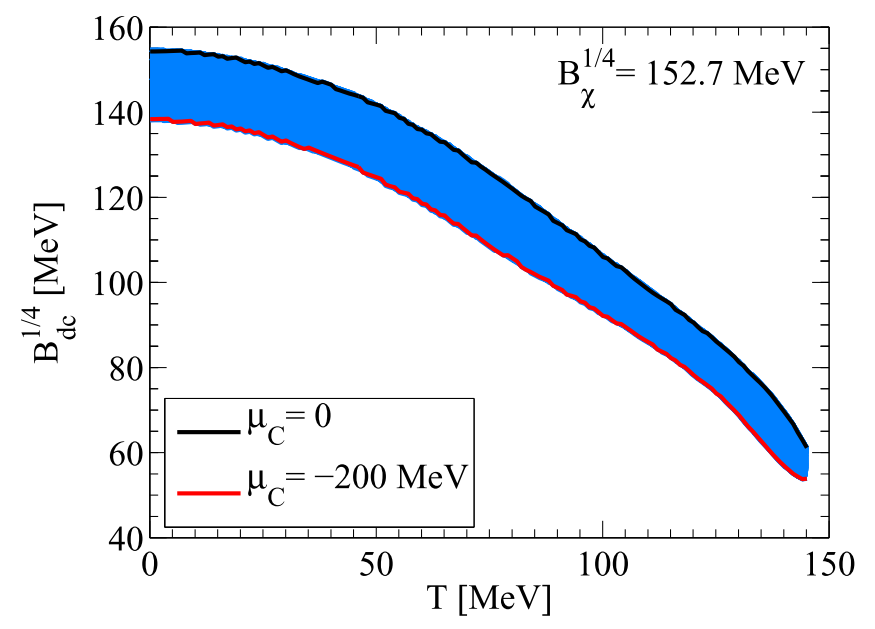

Figure 3. Temperature dependence of the deconfinement bag constant for $\mu_{C}=0, \mu_{C}=-200 \mathrm{MeV}$, and intermediate values (blue band). 


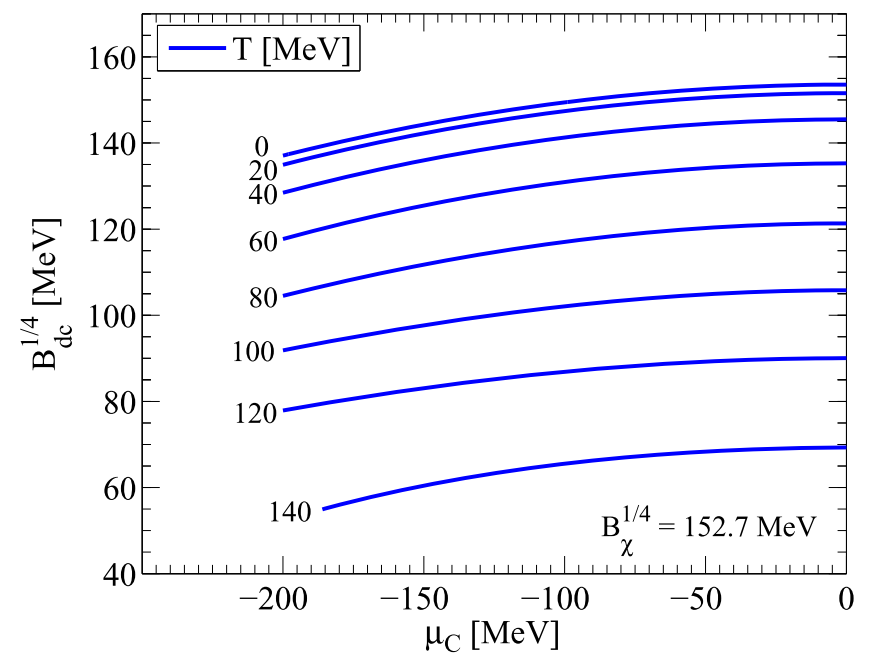

Figure 4. Deconfinement bag constant with respect to temperature $T$ and charge chemical potential $\mu_{C}$.

potential. For any choice of $B_{\chi}$ (we discuss the model dependence on $B_{\chi}$ in Section 5), $B_{\mathrm{dc}}$ decreases with increasing temperature. Simultaneously, the critical baryochemical potential for the phase transition decreases. The related critical temperature where the phase transition line hits the temperature axis is indicated by filled circles in Figure 5, which also shows how it depends on $B_{\chi}$.

By construction, $B_{\mathrm{dc}}$ corresponds to the pressure of the hadronic and quark phase at the phase transition. Therefore, Figures 3 and 5 as well as Figure 4 can also be interpreted as pressure-temperature, respectively pressure-charge chemical potential phase diagrams. The fact that $B_{\mathrm{dc}}$ is decreasing with temperature implies $d P /\left.d T\right|_{P T, \mu_{C}}<0$, which is the opposite of its behavior in the nuclear liquid-gas phase transition, sometimes called an "entropic" phase transition (cf. Hempel et al. 2013; Iosilevskiy 2013, Iosilevskiy 2015 and references therein). This property could lead to interesting consequences for neutron stars and core-collapse supernovae (Hempel et al. 2016; Yudin et al. 2016).

According to our interpretation, $B_{\mathrm{dc}}$ is a measure of the binding energy of quarks in confined hadrons. The decrease of this quantity with increasing temperature indicates nicely that the system indeed becomes less bound. Of course, the Maxwell-like approach and resulting first-order transition are not an appropriate choice in this domain where a crossover transition is expected. This happens in the vicinity of the critical temperature $150<T_{c}<170 \mathrm{MeV}$ (cf. Bazavov et al. 2012a, 2012b; Borsányi et al. 2012, 2014). Our model is not designed to describe a crossover phase transition, and for this reason should not be applied at temperatures where one would expect the critical end point and beyond. However, the drastic decrease of $B_{\mathrm{dc}}$ with increasing temperature (it is rather insensitive to the isospin asymmetry as we illustrate in Figure 4) confirms our interpretation of $B_{\mathrm{dc}}$ as confining binding energy.

Although derivative terms due to the isospin asymmetry and temperature dependence of $B_{\mathrm{dc}}$, Equations (14) and (15), respectively, play no role in determining the phase transition surface (in terms of $\mu_{B}, \mu_{C}$, and $T$ ), they enter the EoS as they contribute to charge density (Equation (12)), entropy (Equation (13)), and energy density (Equation (16)). For the total energy density of quark matter, we introduced $\varepsilon^{\mathrm{Q}}=\tilde{\varepsilon}^{\mathrm{Q}}+B_{\mathrm{dc}}+\varepsilon_{\mathrm{dc}}$, where $\varepsilon_{\mathrm{dc}}$ accounts for all medium-dependent corrections due

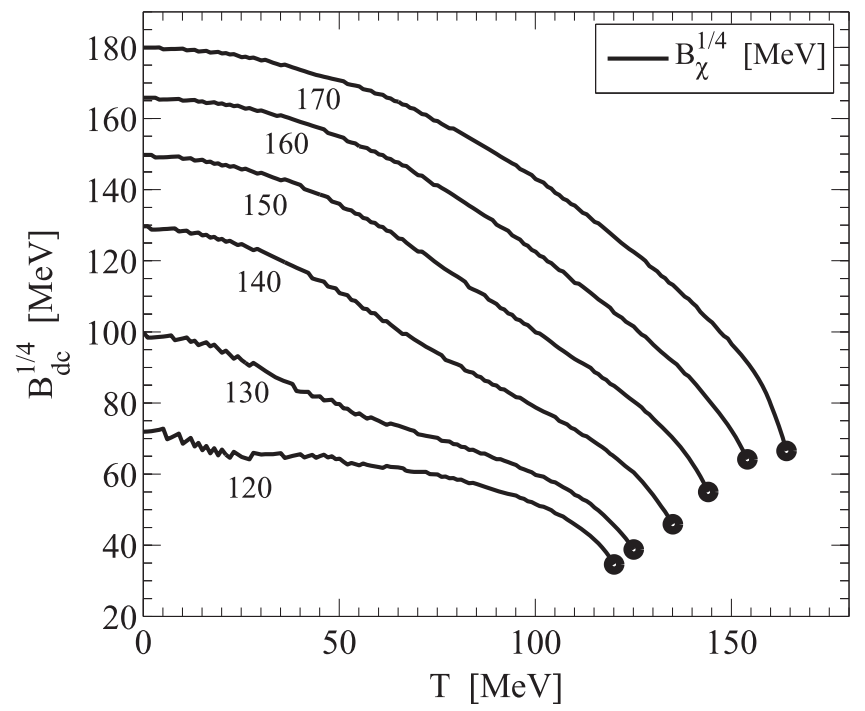

Figure 5. Temperature dependence of the deconfinement bag constant for varying chiral bag constants $B_{\chi}$ in symmetric matter $\left(\mu_{C}=0\right)$.

to derivative terms of $B_{\mathrm{dc}}$. We define the resulting relative deviation on the phase transition hypersurface as $R_{\varepsilon}=\varepsilon_{\mathrm{dc}} / \varepsilon^{\mathrm{Q}}$. These correction terms become smaller when going to higher values of $\mu_{B}>\mu_{B, \chi}$, and go to zero for $\mu_{B} \rightarrow \infty$; therefore $R_{\varepsilon}$ represents the maximal contribution of the correction terms to the energy density. The upper-left panel of Figure 6 illustrates that it is always negative and that the magnitude of these corrections is relatively small compared to the dominant direct contribution of $B_{\mathrm{dc}}$ to $\varepsilon^{\mathrm{Q}}$. As expected, at low temperatures and small isospin asymmetry $R_{\varepsilon}$ turns negligibly small. Even with increasing temperature and isospin asymmetry, it remains small with values up to a magnitude of $10 \%$. However, the opposite is true for derivative corrections to the entropy and charge fraction. The top-right panel of Figure 6 shows the relative contribution of the derivative term of $B_{\mathrm{dc}}$ with respect to $T$ to the total entropy density, $R_{s}=s_{\mathrm{dc}} / s^{\mathrm{Q}}$, which is always negative. Although this quantity can reach a value of -1 at small temperatures, thus reducing the total entropy by a factor of one, one has to keep in mind that at low temperature the entropy is small and hence this is a large relative modification of a quantity with a small value. However, at intermediate temperatures, the changes in the entropy due to the medium dependence of $B_{\mathrm{dc}}$ are far from being negligible. The situation turns even more extreme for the charge density $n_{C}^{\mathrm{Q}}=\tilde{n}_{C}^{\mathrm{Q}}+n_{C, \mathrm{dc}}$ or the corresponding charge fractions $Y_{C}^{\mathrm{Q}}=n_{C}^{\mathrm{Q}} / n_{B}^{\mathrm{Q}}=\tilde{Y}_{C}^{\mathrm{Q}}+Y_{C, \mathrm{dc}}$. The bottom-left panel of Figure 6 illustrates that derivative corrections vanish for values of $\mu_{C}$ close to zero. However, with more negative values of $\mu_{C}$, the correction term $Y_{C \text {,dc }}$ can reach values of up to 0.5 . For the total charge fraction (bottom-right panel), this results in significant changes, generally shifting the charge fraction toward more positive values.

\section{Protoneutron Stars}

We develop the presented model to investigate possible signatures of the QCD phase transition during simulations of core-collapse supernovae (cf. Nakazato et al. 2008; Sagert et al. 2009; Fischer et al. 2011). Such a transition is expected at high baryon densities, $n_{B}>n_{S}$, in the core of a newly born protoneutron star. The latter originates from a core-collapse 

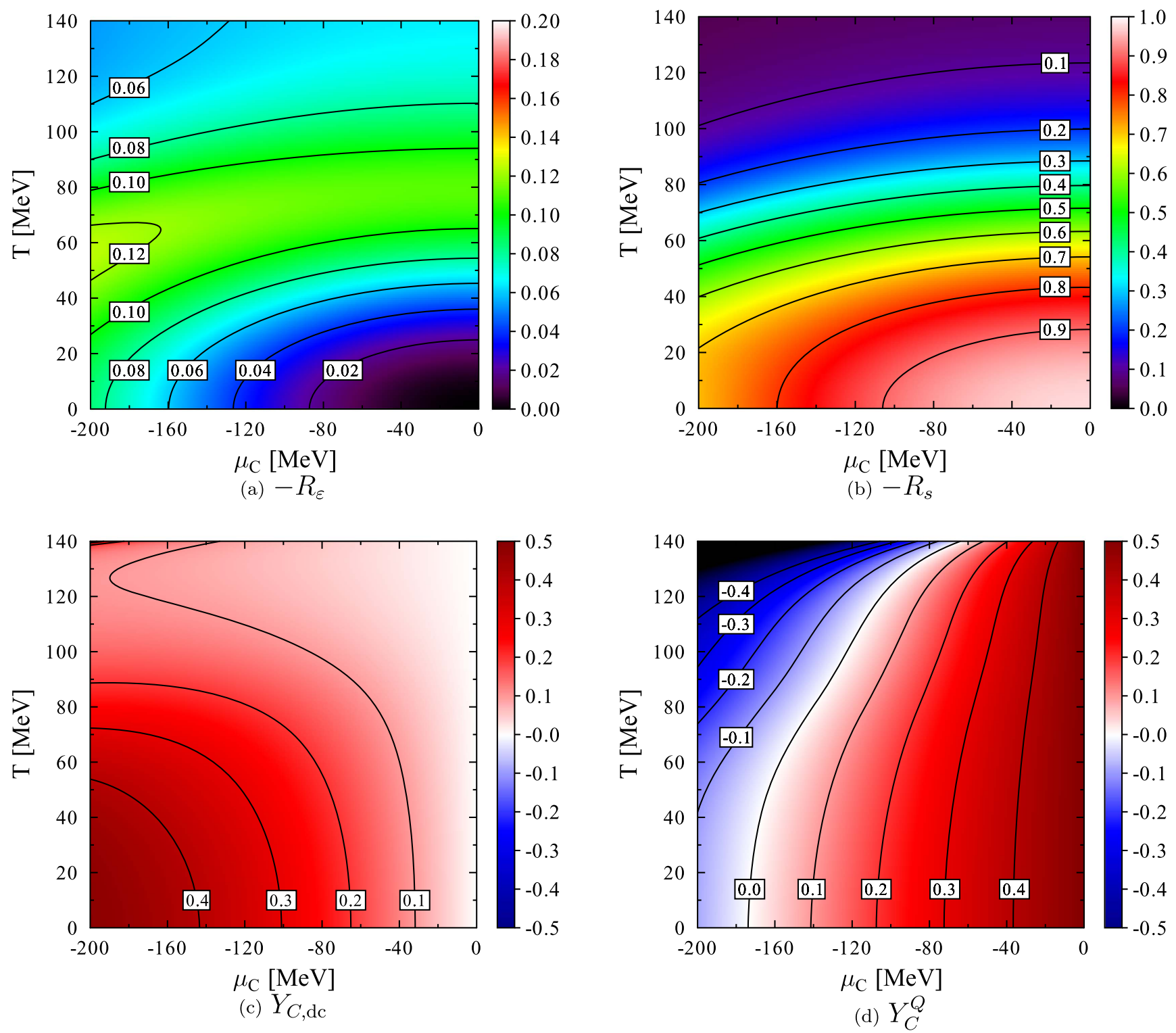

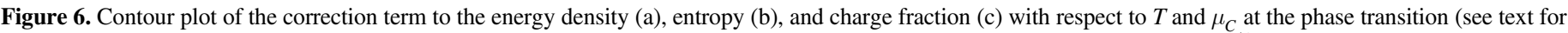
definitions). (d) Corresponding total charge fraction of the quark phase. Notice that negative values of $R_{s}$ and $R_{\varepsilon}$ are shown. $B_{\chi}^{1 / 4}=152.7 \mathrm{MeV}$.

supernova, being triggered from the initially imploding stellar core of a massive star leading finally to the ejection of the stellar mantle, known as the supernova problem.

We first adjust our model such that a cold neutron star can form a quark matter core. The electron gas component in $\beta$ equilibrium is included under the assumption of global charge neutrality. In such a case, they represent a uniform background, which does not influence the phase transition. As pointed out in Klähn \& Fischer (2015), this requires repulsive vector interactions as provided by $v B a g$ in order to describe a maximum neutron star mass of about $2 M_{\odot}$, which is in agreement with the results of Antoniadis et al. (2013) and Fonseca et al. (2016). The inset of Figure 7 shows that vBag provides such massive neutron stars for a vector coupling constant of $K_{v}>2 \times 10^{-6} \mathrm{MeV}^{-2}$. In order to mimic a typical temperature profile for "hot" protoneutron stars we assume an isentropic EoS at two different values for the entropy per particle $\left(s=1,2 k_{B}\right)$. In Figure 7 we show the resulting massradius relations and indicate the core temperature at three characteristic points. In particular, one observes that the quark matter core tends to be significantly cooler than the corresponding core of a purely hadronic star. This is a direct result of the entropic phase transition to quark matter, which has a smaller value of $s$ at any given point in the phase diagram, compared to the nuclear EoS. We further point out that the chosen chiral bag constant of $B_{\chi}^{1 / 4}=152.7 \mathrm{MeV}$ results in a transition density of approximately three times the saturation density in symmetric matter, cf. Figure 8 (lower panel). Although the model allows different choices and correspondingly different transition densities, we consider this value as an acceptable estimate for the lower limit on a possible phase transition to quark matter.

The study of possible consequences of the quark-hadron phase transition based on vBag, for the supernova dynamics as well as on potential observables, e.g., the neutrino and gravitational wave signals, extends beyond the scope of the present paper. This will be explored in an upcoming article. 


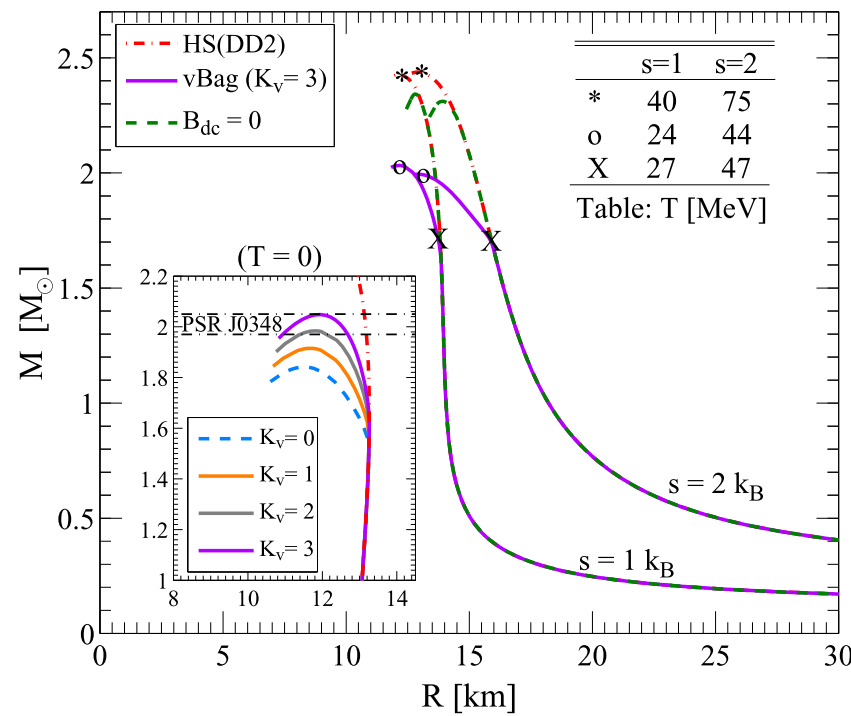

Figure 7. Mass-radius relations for constant entropy per particle $\left(s=1,2 k_{B}\right)$ for $B_{\chi}^{1 / 4}=152.7 \mathrm{MeV}$. The temperature of the most massive NS without a quark core $\left(^{*}\right)$ is in general much larger than the corresponding value for an NS with a quark core (o). By construction, the temperatures in the transition point $(\mathrm{X})$ for the hadron and mixed phases are equal.
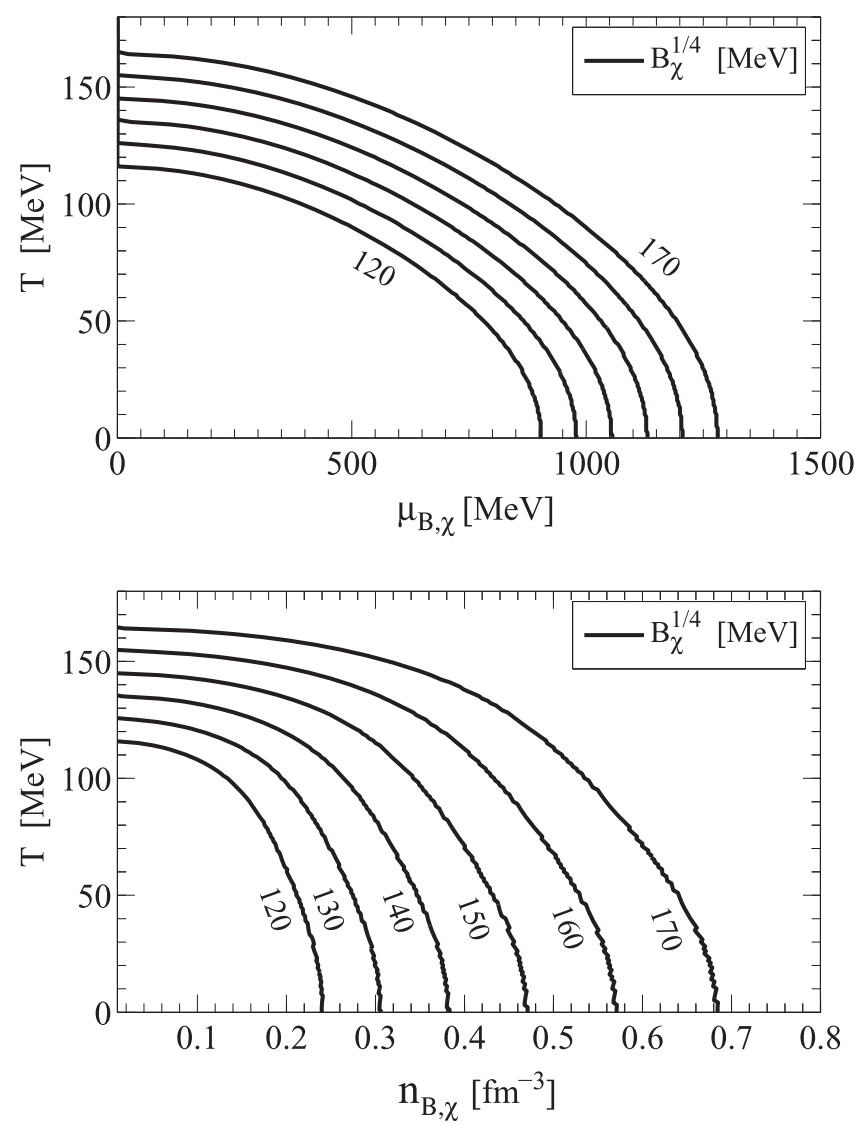

Figure 8. Upper panel: symmetric matter phase diagram for varying chiral bag constants $B_{\chi}$. Bottom panel: corresponding onset density for pure quark matter.

\section{Model Limitations}

We put forward that the deconfinement bag constant has a temperature and isospin dependence which is implicitly given via the hadronic EoS. The chiral condensate and therefore the bag constant $B_{\chi}$ should show an explicit temperature

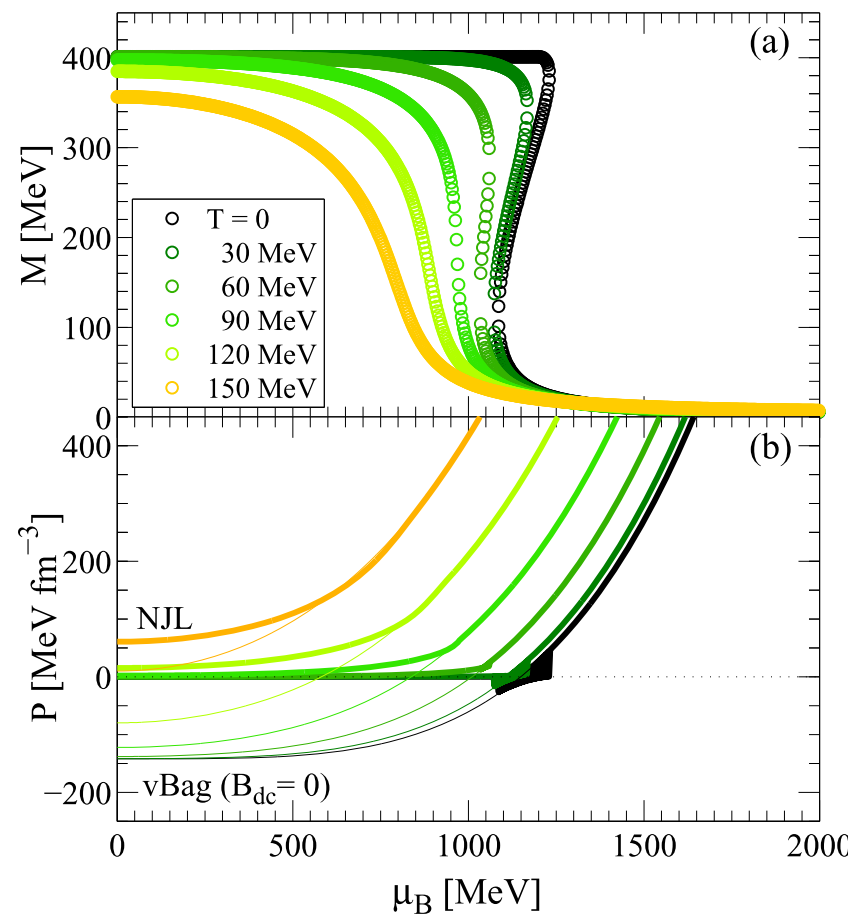

Figure 9. (a) Mass gap solutions of the NJL model used in Klähn \& Fischer (2015) at different temperatures. For temperatures smaller than $90 \mathrm{MeV}$, multiple mass gap solutions exist in the vicinity of the critical quark chemical potential $\mu_{\chi}$. (b) Pressure of the symmetric two-flavor quark matter obtained in the NJL model compared to the ideal gas pressure with bare quark masses at the same temperatures. The ideal gas pressure has been shifted by a temperature-dependent offset to reproduce the high density behavior of the NJL results (details in the text). For temperatures below $90 \mathrm{MeV}$ this shift is nearly constant.

dependence as well. We neglect such dependencies and assume the same fixed value for $B_{\chi}$ at all $T$ and study the impact of varying conditions for the chiral transition to construct our phase diagrams. Figure 8 illustrates that with decreasing $B_{\chi}$, $\mu_{B, \chi}$ and $n_{B, \chi}$ will also decrease substantially. It is formally not difficult to rewrite our approach for a fully medium-dependent chiral bag constant $B_{\chi}$. However, modeling a meaningful medium dependence requires a more elaborate approach than our phenomenological model. We therefore leave this important issue for future investigations and instead provide a brief discussion of the validity of our approach. Before we proceed, we emphasize that we understand vBag to be an extension of the tdBag model rather than an NJL model parameterization even though the extensions are motivated from an NJL model perspective. In this sense all limitations we will discuss in the following hold in a similar way for vBag and tdBAG.

In Klähn \& Fischer (2015) we illustrated that vBag at zero temperature can be parameterized to reproduce the EoS of an NJL model including the typical first-order phase transition in good approximation. Figure 9(a) illustrates that at temperatures larger than $90 \mathrm{MeV}$, only one mass gap solution exists at any given quark chemical potential and thus no distinct first-order phase transition is obtained. For temperatures beyond this value, one additionally observes a decrease of the mass gap already at zero quark chemical potential.

This behavior of the dressed mass $M$ at $\mu=0$ is shown for a wider range of temperatures in Figure 10. Additionally, we plot the difference of the pressure of light quarks with temperaturedependent dressed mass to the pressure of bare mass quarks at 


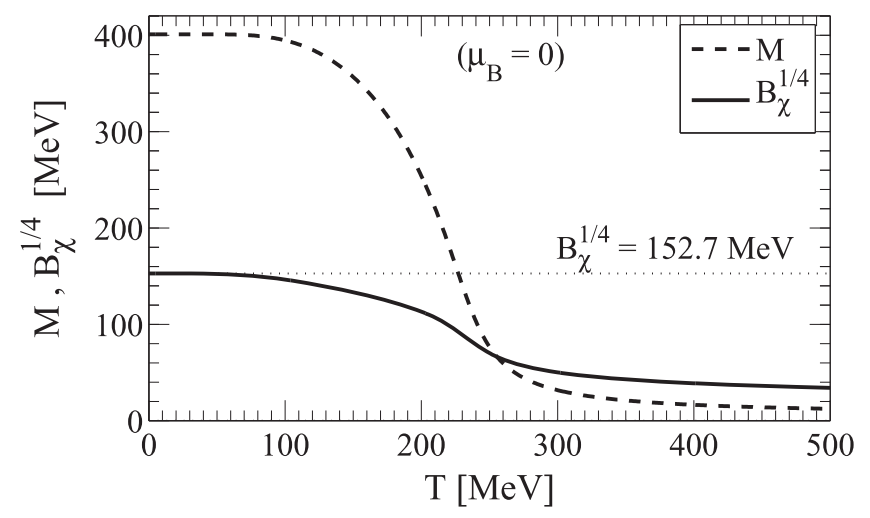

Figure 10. Temperature dependence of the mass gap solutions of the NJL model used in Klähn \& Fischer (2015) at zero quark chemical potential and the resulting one flavor chiral bag constant.

$\mu=0$. In both cases, the vacuum pressure according to Equation (17) in Klähn \& Fischer (2015) is included. The temperature dependence up to $100 \mathrm{MeV}$ is negligible.

Figure 9(b) shows the full dependence of the pressure on the chemical potential for different temperatures as we obtain it within the NJL model, considering only the scalar contributions to the self energy (thick lines). The increasing offset at $\mu=0$ with increasing temperature results from the thermal excitation of quarks and antiquarks. We further plot the corresponding results for an ideal gas of quarks with undressed bare mass (thin lines). This corresponds to the vBag model with $K_{v}=0$. The offset between the NJL and vBag results is determined at zero $\mu$ in the same way we determine $B_{\chi}$; however for this plot, it is determined at finite temperature. Figure 10 shows this quantity. Again, the difference from the $T=0$ result is small for temperatures below about $90 \mathrm{MeV}$. Moreover, Figure 9(b) shows that at sufficiently large chemical potential, both approaches give the same result at all temperatures. Of course, this is the same behavior one observes at $T=0$ and which initially motivated us to introduce vBag as a simplified model EoS that does not require solving gap equations explicitly.

In conclusion, although not intended to fully mimic NJL model calculations, vBag sufficiently reproduces the NJL results for temperatures up to $90 \mathrm{MeV}$. Higher temperatures already correspond to conditions that are typically out of reach for astrophysical applications, e.g., core-collapse supernovae and neutron star scenarios. Finally, we remark that neither NJL nor vBag nor the hadronic EoS HS(DD2) are developed for large temperatures and small chemical potentials, e.g., to study the QCD phase transition as in particular in the transition domain thermally excited mesons (e.g., pions) and additional nuclear resonances have to be taken into account.

Moreover, neither strange quarks nor hyperons are considered in this study. It is a worthwhile question to investigate whether the transition to quark matter happens before or after hyperon degrees are excited. However, as little is known about hyperon interactions, we once again would have to make model assumptions that crucially affect the answer to this question, cf. Bonanno \& Sedrakian (2012) and Masuda et al. (2016).

\section{Summary and Discussions}

In this article, we introduced an important extension of the recently introduced quark matter EOS vBag (for details, see Klähn \& Fischer 2015) to finite temperature and arbitrary isospin asymmetry. As an extension of tdBag, it takes $\mathrm{D} \chi \mathrm{SB}$ explicitly into account in terms of the chiral bag constant $B_{\chi}$. It imposes the restoration of chiral symmetry at an associated critical chemical potential $\left(\mu_{B, \chi}\right)$. This first model parameter is complemented by a second one, $K_{v}$, which is the vector coupling constant due to repulsive vector interactions-not included in tdBag-which stiffens the EoS with increasing density. The last main characteristics of vBag is the inherent connection to a given hadronic EoS in terms of the mediumdependent deconfinement bag constant $\left(B_{\mathrm{dc}}\right)$. It is determined entirely by the chiral transition and a selected hadronic EoSand in this sense is not a free parameter of vBag. With this procedure we assure that chiral and deconfinement phase transitions coincide, assuming that the transition is of first order. This purely phenomenologically introduced medium dependence of the deconfinement bag constant of vBag to the hadronic EoS based on the assumption of simultaneous chiral symmetry restoration and deconfinement. In this sense, our approach differs from quark models that aim to determine the medium dependence of a bag constant microscopically (cf. Roberts \& Schmidt 2000 and Buballa 2005 and references therein for a discussion of NJL and DS analyses, respectively).

With vBag and the extension to finite temperatures and isospin asymmetry, we provide a novel quark matter EoS for a broad spectrum of applications in astrophysics, e.g., simulations of neutron star mergers, core-collapse supernovae, and protoneutron star cooling, where commonly the simple tdBag model has been employed (cf. Pons et al. 2001; Nakazato et al. 2008; Sagert et al. 2009; Fischer et al. 2011, and references therein). tdBag does not account for chiral physics, and without perturbative corrections violates the $2 M_{\odot}$ neutron star mass constraint, due to missing repulsive interactions. Here, vBag provides solutions that cure both problems without losing the simplicity and flexibility of tdBag.

A natural consequence of the extension to finite temperatures and isospin asymmetry is the emergence of an implicit temperature and isospin asymmetry dependence of the deconfinement bag constant, $B_{\mathrm{dc}}\left(T, \mu_{C}\right)$. It is determined by the pressure of the hadronic EoS at the chiral transition. In addition, corrections to entropy and charge density arise from a thermodynamically consistent treatment. The phase transition surface in the phase diagram (in terms of $\mu_{B}, \mu_{C}$, and $T$ ) is independent of these correction terms. Furthermore, the resulting corrections to the energy density are small while we find significant contributions to the entropy density and charge fraction of quark matter. Overall, we find that simultaneous chiral symmetry restoration and deconfinement make the quark phase more similar to the hadronic phase in terms of its temperature and asymmetry dependence.

The hybrid EoS is constructed based on our choice of the nuclear RMF EoS HS(DD2). Starting from the assumption $\mu_{B, \mathrm{dc}}=\mu_{B, \chi}$, the phase boundary in the phase diagram shows distinct differences from the common approach used in astrophysics. The standard procedure results in significantly higher transition densities than our approach with finite $B_{\mathrm{dc}}$. Moreover, we find a different behavior of the phase boundary toward increasing temperatures. The coexistence region between the onset of quark matter and pure quark matter broadens, unlike in the "standard" approach. Since the deconfinement bag constant maps the pressure of the hadronic EoS at the transition line, its medium dependence is strongly affected by the hadronic EoS. It decreases with increasing temperature and rather weakly depends on the isospin 
asymmetry. Furthermore, we briefly discussed the expected impact of a temperature-dependent chiral bag constant by choosing different values of $B_{\chi}$. The phase boundaries shift to lower (higher) densities for lower (larger) values of $B_{\chi}$. This important aspect will be further explored in future studies, as it is beyond the scope of the present article.

At temperatures in excess of about $100 \mathrm{MeV}$, we are leaving the domain of validity of our purely nucleonic EoS; nuclear resonances and mesons such as pions are not explicitly included in the description of the hadronic medium of HS(DD2). Nevertheless, the qualitative behavior of the vBag phase boundary seems to be in agreement with predictions of lattice-QCD and data from heavy-ion collision experiments with one important exception: the phase transition we describe in our approach is by construction always of first order.

The authors acknowledge support from the Polish National Science Center (NCN) under grant numbers DEC-2011/02/A/ ST2/00306 (T.F.) and UMO-2013/09/B/ST2/01560 (T.K.). M.H. acknowledges support from the Swiss National Science Foundation. Partial support comes from "NewCompStar," COST Action MP1304.

\section{References}

Andronic, A., Blaschke, D., Braun-Munzinger, P., et al. 2010, NuPhA, 837, 65 Antoniadis, J., Freire, P. C. C., Wex, N., et al. 2013, Sci, 340, 448 Aoki, Y., Endrodi, G., Fodor, Z., Katz, S., \& Szabo, K. 2006, Natur, 443, 675 Barz, H. W., Friman, B. L., Knoll, J., \& Schulz, H. 1989, PhRvD, 40, 157 Bazavov, A., Bhattacharya, T., Cheng, M., et al. 2012a, PhRvD, 85, 054503 Bazavov, A., Ding, H.-T., Hegde, P., et al. 2012b, PhRvL., 109, 192302 Bazavov, A., et al. 2014, PhRvD, 90, 094503

Blaschke, D., Sandin, F., Klahn, T., \& Berdermann, J. 2009, PhRvC, 80, 065807

Bonanno, L., \& Sedrakian, A. 2012, A\&A, 539, A16

Borsányi, S., Fodor, Z., Hoelbling, C., et al. 2014, PhLB, 730, 99

Borsányi, S., Fodor, Z., Katz, S. D., et al. 2012, JHEP, 1, 138

Braun-Munzinger, P., Kalweit, A., Redlich, K., \& Stachel, J. 2015, PhLB, 747, 292

Buballa, M. 2005, PhR, 407, 205

Chen, H., Baldo, M., Burgio, G., \& Schulze, H.-J. 2011, PhRvD, 84, 105023
Chen, H., Wei, J.-B., Baldo, M., Burgio, G. F., \& Schulze, H.-J. 2015, PhRvD, 91, 105002

Chen, H., Wei, J. B., \& Schulze, H. J. 2016, EPJA, 52, 291

Chen, H., Yuan, W., Chang, L., et al. 2008, PhRvD, 78, 116015

Demorest, P. B., Pennucci, T., Ransom, S. M., Roberts, M. S. E., \& Hessels, J. W. T. 2010, Natur, 467, 1081

Dexheimer, V. A., \& Schramm, S. 2010, PhRvC, 81, 045201

Farhi, E., \& Jaffe, R. 1984, PhRvD, 30, 2379

Fischer, T., Sagert, I., Pagliara, G., et al. 2011, ApJS, 194, 39

Fodor, Z., \& Katz, S. 2004, JHEP, 0404, 050

Fonseca, E., Pennucci, T. T., Ellis, J. A., et al. 2016, ApJ, 832, 167

Grigorian, H. 2007, PPNL, 4, 223

Hempel, M., Dexheimer, V., Schramm, S., \& Iosilevskiy, I. 2013, PhRvC, 88, 014906

Hempel, M., Heinimann, O., Yudin, A., et al. 2016, PhRvD, 94, 103001

Hempel, M., \& Schaffner-Bielich, J. 2010, NuPhA, 837, 210

Iosilevskiy, I. 2013, in Physics of Extreme States of Matte, ed. V. Fortov et al (Chernogolovka, Russia: IPCP RAS Publishing)

Iosilevskiy, I. 2015, in Compact Stars in the QCD Phase Diagram IV (CSQCDIV), ed. D. Blaschke \& T. Fischer (Prerow, Germany) C140926

Klähn, T., \& Fischer, T. 2015, ApJ, 810, 134

Klähn, T., Roberts, C. D., Chang, L., Chen, H., \& Liu, Y.-X. 2010, PhRvC, 82, 035801

Klevansky, S. 1992, RvMP, 64, 649

Kojo, T., Powell, P. D., Song, Y., \& Baym, G. 2015, PhRvD, 91, 045003

Kurkela, A., Fraga, E. S., Schaffner-Bielich, J., \& Vuorinen, A. 2014, ApJ, 789, 127

Masuda, K., Hatsuda, T., \& Takatsuka, T. 2013, PTEP, 2013, 070003

Masuda, K., Hatsuda, T., \& Takatsuka, T. 2016, EPJA, 52, 65

McLerran, L., \& Pisarski, R. D. 2007, NuPhA, 796, 83

McLerran, L., Redlich, K., \& Sasaki, C. 2009, NuPhA, 824, 86

Nakazato, K., Sumiyoshi, K., \& Yamada, S. 2008, PhRvD, 77, 103006

Nambu, Y., \& Jona-Lasinio, G. 1961, PhRv, 122, 345

Pagliara, G., \& Schaffner-Bielich, J. 2008, PhRvD, 77, 063004

Pons, J. A., Steiner, A. W., Prakash, M., \& Lattimer, J. M. 2001, PhRvL, 86,5223

Rischke, D. H., Friman, B. L., Stocker, H., \& Greiner, W. 1988, JPhG, 14, 191

Roberts, C. D., \& Schmidt, S. M. 2000, PrPNP, 45, S1

Sagert, I., Fischer, T., Hempel, M., et al. 2009, PhRvL, 102, 081101

Steinheimer, J., Schramm, S., \& Stöcker, H. 2011, JPhG, 38, 035001

Typel, S., Oertel, M., \& Klähn, T. 2015, PPN, 46, 633

Typel, S., Röpke, G., Klähn, T., Blaschke, D., \& Wolter, H. 2010, PhRvC, 81, 015803

Yasutake, N., Łastowiecki, R., Benić, S., et al. 2014, PhRvC, 89, 065803

Yudin, A. V., Hempel, M., Nadyozhin, D. K., \& Razinkova, T. L. 2016, MNRAS, 455, 4325 